\title{
A novel homozygous ALMS1 protein truncation mutation (c.2938dupA) revealed variable clinical expression among Saudi Alström syndrome patients
}

\author{
Amnah Yousuf Bdier ${ }^{1}$, Faten Abdullah Al-Qahtani ${ }^{2,3}$, Prashant Kumar Verma ${ }^{1,2}$, \\ Naeem Abdulmoneem Alshoaibi ${ }^{4}$, Nuha Mohammed Alrayes ${ }^{5}$, Noor Ahmad Shaik ${ }^{1,2}$, \\ Roger Sik Yin Foo ${ }^{4}$, Zahurul Alam Bhuiyan ${ }^{6}$, Jumana Yousuf Al-Aama ${ }^{1,2}$
}

\author{
1Princess Al Jawhara Albrahim Center of Excellence in Research of Hereditary \\ Disorders, King Abdulaziz University, Jeddah, Saudi Arabia \\ ${ }^{2}$ Department of Genetic Medicine, King Abdulaziz University, Jeddah, Saudi Arabia \\ ${ }^{3}$ Department of Clinical Laboratory Science, Prince Sultan Military College of Health \\ Science, Dammam, Saudi Arabia \\ ${ }^{4}$ Cardiology Department of Medicine, King Abdulaziz University, Jeddah,Saudi Arabia \\ ${ }^{5}$ Medical Laboratory Technology, Faculty of Applied Medical Sciences, King Abdulaziz \\ University, Jeddah, Saudi Arabia \\ ${ }^{6} U$ nité de Recherche Cardiogénétique, Service de Médecine Génétique, Centre \\ Hospitalier Universitaire Vaudois (CHUV), Lausanne, Switzerland
}

Submitted: 12 May 2020

Accepted: 8 September 2020

Arch Med Sci

DOI: https://doi.org/10.5114/aoms.2020.100635

Copyright $\odot 2020$ Termedia \& Banach

\begin{abstract}
Introduction: Alström syndrome, ALMS (OMIM 203800) is a rare multi-systemic disease. The characteristic clinical features include blindness due to progressive cone-rod dystrophy, sensorineural hearing loss, type 2 diabetes mellitus, dilated cardiomyopathy, and childhood obesity. The aim of this study was to identify the genetic cause of Alström syndrome in patients who presented with variable clinical characteristics.

Material and methods: Clinical phenotyping and whole exome sequencing were performed in Saudi Alström syndrome patients. The Sanger sequencing was done to ascertain the segregation of Alström syndrome causative mutation in the family members. The rare prevalence of this mutation was further established by sequencing an additional 100 healthy Saudi controls. Results: Whole exome sequencing analysis revealed that Alström syndrome patients have inherited a novel homozygous protein truncating mutation (c.2938dupA) in the ALMS1 gene segregated in an autosomal recessive fashion. This variant was absent in healthy controls. Genotype-phenotype analysis showed its interesting association with intra-familial clinical variability with regards to vision abnormalities, age at onset of dilated cardiomyopathy (DCM), obesity and hearing loss symptoms in the Alström syndrome patients. Conclusion: Our findings indicate that the atypical presentation of Alström syndrome, even within siblings, could sometimes lead to clinical misdiagnosis. Hence, the present study emphasizes the utility of exome sequencing to support the clinical diagnosis of Alström syndrome patients.
\end{abstract}

Key words: Alström syndrome, ALMS1, phenotypic variability, protein truncating mutation.
Corresponding authors: Faten Abdullah Al- Qahtani E-mail: faalqahtani44@gmail.com Dr. Jumana Yousuf Al-Aama E-mail: jalama@kau.edu.sa 
Amnah Yousuf Bdier, Faten Abdullah Al-Qahtani, Prashant Kumar Verma, Naeem Abdulmoneem Alshoaibi, Nuha Mohammed Alrayes, Noor Ahmad Shaik, Roger Sik Yin Foo, Zahurul Alam Bhuiyan, Jumana Yousuf Al-Aama

\section{Introduction}

Alström syndrome, ALMS (OMIM 203800), is a multisystemic disorder found with a rare prevalence of $<1$ per 1,000,000 individuals in the general population [1-3]. Approximately 1200 Alström syndrome cases have been diagnosed worldwide $[1,4,5]$. The main clinical characteristics of this syndrome are sensorineural hearing loss ( $\mathrm{SNHL}$ ), blindness due to progressive cone-rod dystrophy, short stature, type 2 diabetes mellitus (T2DM), and childhood obesity associated with hyperinsulinemia $[6,7]$. Although dilated cardiomyopathy (DCM) is the predominant observation in most Alström syndrome patients, additional clinical manifestations such as renal failure, hepatic, pulmonary and urologic dysfunctions are also known to be evident in them [8]. Most clinical manifestations start in early infancy, but the age of onset and severity of symptoms greatly vary in patient groups [5]. The clinical diagnosis of Alström syndrome is usually established based on the clinical features, medical history and positive family history of the affected patient, but overlapping clinical features makes it more challenging. On the other hand, molecular genetic diagnosis of Alström syndrome not only helps to ascertain the clinical diagnosis but also could assist clinicians in choosing an appropriate therapeutic management strategy.

Alström syndrome is regarded as a classical monogenic disease as it is well established to be caused by pathogenic mutations in the ALMS1 gene, which spans over 23 exons and encodes a 4168 amino acid long protein [2, 5, 9]. ALMS1 protein is expressed in renal tubules, heart, liver, the organ of Corti, retinal photoreceptors, pancreatic islets, and the hypothalamus region of the brain [10]. It plays a critical role in ciliary function and regulates the cell cycle as well as intracellular transport. To date, more than 250 disease-causing mutations in ALMS1 have been reported in Alström syndrome patients $[5,9,11]$. Most of them are nonsense or frame shift mutations resulting in premature mRNAs, which are eventually eliminated by a nonsense mediated decay pathway.

Approximately, $94 \%$ of the ALMS1 mutations have been found in exons 8,10 and $16[12,13]$. So far, the majority of these studies have been published on sporadic Alström syndrome cases [14, 15]. However, studying familial cases has the potential not only to inform about the most affected organ system and clinical spectrum but could also shed light on the molecular basis of the disease. In Saudi Arabia, the average rate of consanguinity is $50 \%$, but in certain provinces it even reaches $80 \%$. Consanguinity enriches the presence of defective alleles in any population and acts as a major underlying factor for several genetic disorders, especially populationspecific autosomal recessive disorders [16]. Hence, studying the familial form of Alström syndrome in Saudi patients provides an advantage both to examine the founder effect of ALMS1 mutations and also assess its impact on clinical variability among the family members. Therefore, in this prospective study, we performed whole exome sequencing of Saudi Alström syndrome patients and identified the disease causative mutation, determined its segregation pattern and analyzed the phenotypic expression of the disease in the family members carrying the same mutation.

\section{Material and methods}

\section{Ethical approval}

All adult participants of this study have provided written informed consent, but for underage participants (children below 18 years ages) consent was obtained from their parents. The ethical approval for the present study was obtained by the Unit of Biomedical Ethics Research Committee, Faculty of Medicine, King Abdulaziz University, Jeddah.

\section{Study subjects}

A Saudi family with two affected patients presenting frequent heart palpitations was recruited from the cardiogenetics clinic of Princess Al-Jawhara Al-Brahim Center of Excellence in Research of Hereditary Disorders (PACER-HD). These two affected siblings underwent detailed clinical examinations including biochemical investigations, electrocardiogram, echocardiography, abdominal ultrasonography, chest radiograph, audiometry and visual evoked potentials. The full clinical history of the family members was collected during their regular visits. We also recruited 100 healthy Saudi controls, who have no history of genetic diseases in their respective families.

\section{Sampling}

Peripheral blood samples $(2-5 \mathrm{ml})$ from all the participants were collected in K3 EDTA tubes (BD Vacutainer, USA) and stored at $4^{\circ} \mathrm{C}$ until they were analyzed. Genomic DNA was extracted using the Qiagen DNA Extraction Mini Kit (QIAamp mini kit, USA). The DNA purity and concentration were determined using the NanoDrop 2000/2000c Spectrophotometer (Thermo-Scientific, USA).

\section{Exome sequencing}

Whole exome sequencing (WES) was performed for the proband (II.2) and his brother (II.5) at a local commercial facility. The genomic DNA was subjected to bidirectional exome sequencing at $100 \times$ resolution. DNA libraries were prepared using the Sureselect target enrichment system 
(Agilent Technologies, USA) from $2 \mu \mathrm{g}$ of genomic DNA of the patients. The enriched libraries with 90 base-pair long reads were then sequenced on the Illumina HiSeq2500 platform (Illumina, USA). The raw sequencing data were obtained in the form of FASTQ files. Low quality read sequences were discarded. The short sequences generated in the WES were lined up to a reference genome (UCSC hg19) (http://genome.ucsc.edu/) with the Burrows-Wheeler Alignment (BWA) tool. Highquality single nucleotide variants (SNVs) and Indels detection was performed using GATKv3.0 Haplotype Caller and cross checked against sequences in the 1000 Genomes Project databases (http://www.1000genomes.org/data) and the dbSNP (http://www.ncbi.nlm.nih.gov/snp/). Variants were prioritized in a stepwise manner following the data analysis pipeline (Figure 1). Since mutations in the ALMS1 gene are known to be causative for Alström syndrome, we initially checked the deleterious variants in this gene. We searched for those ALMS1 variants which are extremely rare (minor allele frequency MAF $<0.001$ ) and occur in the coding (missense, frameshift) and regulatory (splice site and promoter) regions.

We determined the rare frequency or novelty of ALMS1 variants by comparing them in different databases such as 1000 Genomes Project (http:// browser.1000genomes.org/index.html), the Exome Aggregation Consortium (ExAC) database that includes exome sequencing information for 60,706 persons as part of a disease-specific and population genetic study (http://exac.broadinstitute.org), the Greater Middle East (GME) variome project which is considered as a coding base reference for the countries in the Greater Middle East and includes exome sequencing data for 2,497 individuals (http://igm.ucsd.edu/gme/), the EUROWABB Leiden Open Variation Database (LOVD), which is a locus-specific database for Alström syndrome and other rare diabetes syndromes (https:// databases.lovd.nl/shared/variants/ALMS1/unique) 10, and finally in the Saudi Human Genome Project (SHGP) database, which hosts whole exome sequencing data of 6033 Saudi individuals. The potential ALMS1 variant selected from exome sequencing was screened by the Sanger sequencing method in the family members and in 100 healthy control subjects. The rationale to screen the first-degree relatives (parents and siblings) is to determine the mode of its inheritance in the family, and healthy controls is to ensure its rarity in the population.

\section{Sanger sequencing}

The shortlisted ALMS1 mutation was validated through Sanger sequencing. In the initial steps, primer sets flanking the target gene region were in-house designed and their chemical synthesis

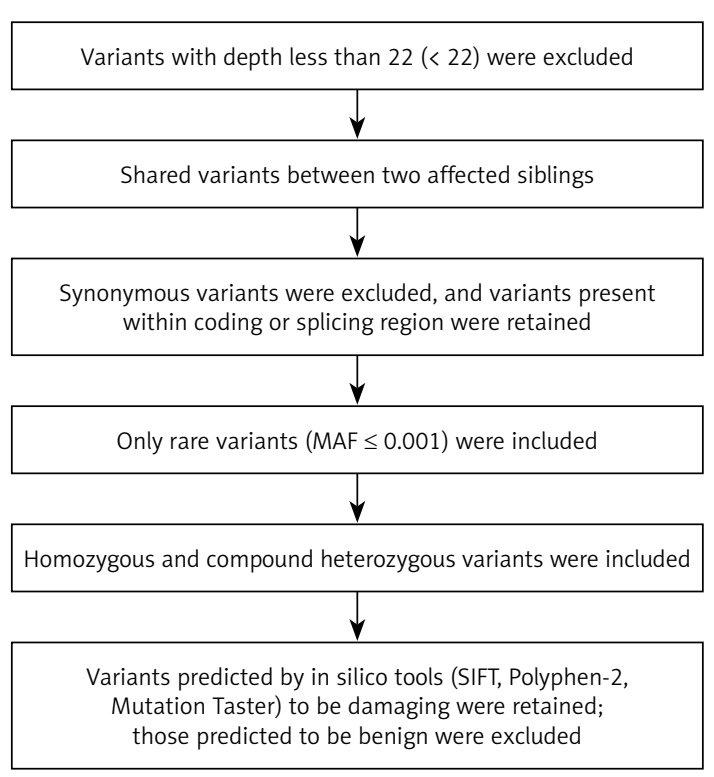

Figure 1. The whole exome sequencing data analysis pipeline used for identification of the ALMS1 variant

was done at a commercial facility. Subsequently, polymerase chain reaction (PCR), gel electrophoresis, PCR purification and cycle sequencing steps were conducted as per standard protocols. PCR amplicons were bidirectionally sequenced using the ABI-Prism 3730xl Genetic Analyzer (Applied Biosystems, USA). The Sanger sequencing files were aligned and compared to the reference sequence via BioEdit sequence alignment editor (version 6.0.7) (www.mbio.ncsu.edu/BioEdit/bioedit.html). Mutation numbering was done by considering $A$ of codon ATG as the first nucleotide of the ALMS1 gene.

\section{Results}

\section{Clinical evaluation}

The proband (II.2) (Figure 2, Table I) in this family is a 22-year-old man born to consanguineous parents. Initially, at the age of 21 , he was suspected to have cardiac arrhythmia, because he showed abnormal electrocardiogram (ECG) findings, palpitations, seizures and repeated vertigo. ECG showed rapid atrial fibrillation (AF). Then, he developed heart failure and was diagnosed with dilated cardiomyopathy. The patient could not tolerate a small dose of $\beta$-blocker and due to the frequent bradycardia and the nonspecific conduction delay of the QRS (around $120 \mathrm{~ms}$ ), the treating physician preferred to implant a cardiac resynchronization therapy (CRT) device and started on anti-heart failure medications (dabigatran (110 mg BID), amiodarone (200 mg OD), spironolactone $(25 \mathrm{mg})$ ). From birth, the proband (II:2) had progressive hearing, vision loss (severe progres- 
Amnah Yousuf Bdier, Faten Abdullah Al-Qahtani, Prashant Kumar Verma, Naeem Abdulmoneem Alshoaibi, Nuha Mohammed Alrayes, Noor Ahmad Shaik, Roger Sik Yin Foo, Zahurul Alam Bhuiyan, Jumana Yousuf Al-Aama

sive retinal dystrophy and cataract) and obesity. Consequently, he was initially suspected to have a mitochondrial disorder. (Note: recently he is on sacubitril/valsartan 49/51 mg BID, bisoprolol $5 \mathrm{mg}$, furosemide $40 \mathrm{mg}$, spironolactone $25 \mathrm{mg}$, acetylsalicylic acid $81 \mathrm{mg}$ ). The treating physician stopped the oral anticoagulant after 6 months, believing that the atrial fibrillation was only one episode and there was no recurrence during device interrogation follow-up. Acetylsalicylic acid $81 \mathrm{mg}$ was taken as an over-the-counter medication by the family without physician recommendation.

The proband (II.2) (Figure 2, Table I) has two brothers; one (II.5) unfortunately died at the age

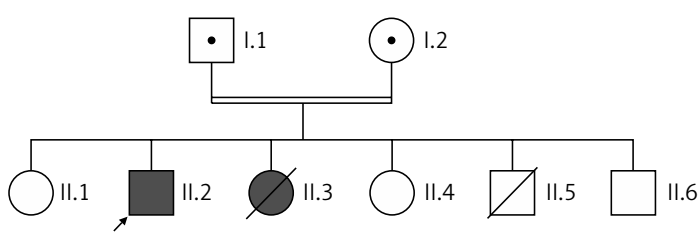

Figure 2. Pedigree analysis of the family, segregating Alström syndrome in autosomal recessive fashion. Circles and squares indicate females and males, respectively. Darkened symbols stand for affected participants, symbols having a dot inside stand for heterozygous carriers. Arrow indicates the proband of 16,6 months after we performed the genetic testing. His ECG showed abnormal positive R wave in V1, ST depression and non-specific diffuse $T$ wave abnormality. He had progressive vison loss from birth like the proband (II.2). However, he had DCM from birth and a few other symptoms such as acanthosis nigricans and endocrine disturbances (adrenal and thyroid glands). The reason for his sudden cardiac death at home most likely was secondary to ventricular arrhythmia. The second 13-year-old brother (II.6) was healthy. He also had 3 sisters; one (II.3) presented with vomiting and lethargy then was diagnosed with Wilms' tumor. She died at the age of 7 years because of complications of tumors. We are not aware of clinical symptoms other than skin allergy. The remaining two sisters (II.1 and II.4) were healthy and did not present any symptoms of the disease. The parents were both apparently healthy (I.1 and I.2).

\section{Mutation analysis}

WES was performed for the proband (II.2) and his brother (II.5). After a series of variant filtration steps, both the proband (II.2) and his brother (II.5) were found to have co-inherited c.2938dupA, a novel frameshifting insertion mutation located in the exon 8 region of the ALMS1 gene in the homozygous form (Figure 3 ). The novelty of this variant was ascertained through its absence in 1000G,

Table I. Clinical features for all members of the family

\begin{tabular}{|c|c|c|c|c|c|c|}
\hline Clinical signs & $\mathrm{I}-1$ & $\mathrm{I}-2$ & II-2 & II-4 & II-5 & II-6 \\
\hline Age of onset of symptoms [years] & - & - & Since birth & - & Since birth & - \\
\hline Age at genetic diagnosis [years] & 59 & 41 & 21 & 16 & 14 & 13 \\
\hline Vision loss & - & - & At puberty & - & At puberty & - \\
\hline Hearing loss & - & - & $\sqrt{ }$ & - & - & - \\
\hline Obesity & - & - & $\sqrt{ }$ & - & - & - \\
\hline Dilated cardiomyopathy (DCM) & - & - & $\sqrt{ }$ & - & $\sqrt{ }$ & - \\
\hline Renal disease & - & - & - & - & - & - \\
\hline $\begin{array}{l}\text { Nystagmus and retinal dystrophy } \\
\text { in both eyes }\end{array}$ & - & - & $\sqrt{ }-\sqrt{ }$ & - & $\sqrt{ }-\sqrt{ }$ & - \\
\hline Acanthosis nigricans & - & - & - & - & $\sqrt{ }$ & - \\
\hline Neuropsychiatric disorders & - & - & $\sqrt{ }$ & - & $\sqrt{ }$ & - \\
\hline Diabetes mellitus (DM) & - & - & - & - & - & - \\
\hline $\begin{array}{l}\text { Endocrine disturbances (adrenal } \\
\text { and thyroid glands) }\end{array}$ & - & - & - & - & $\sqrt{ }$ & - \\
\hline Triglyceridemia & - & - & $\sqrt{ }$ & - & - & - \\
\hline Short stature & - & - & - & - & - & - \\
\hline Allergic rhinitis & - & - & $\sqrt{ }$ & - & - & - \\
\hline
\end{tabular}




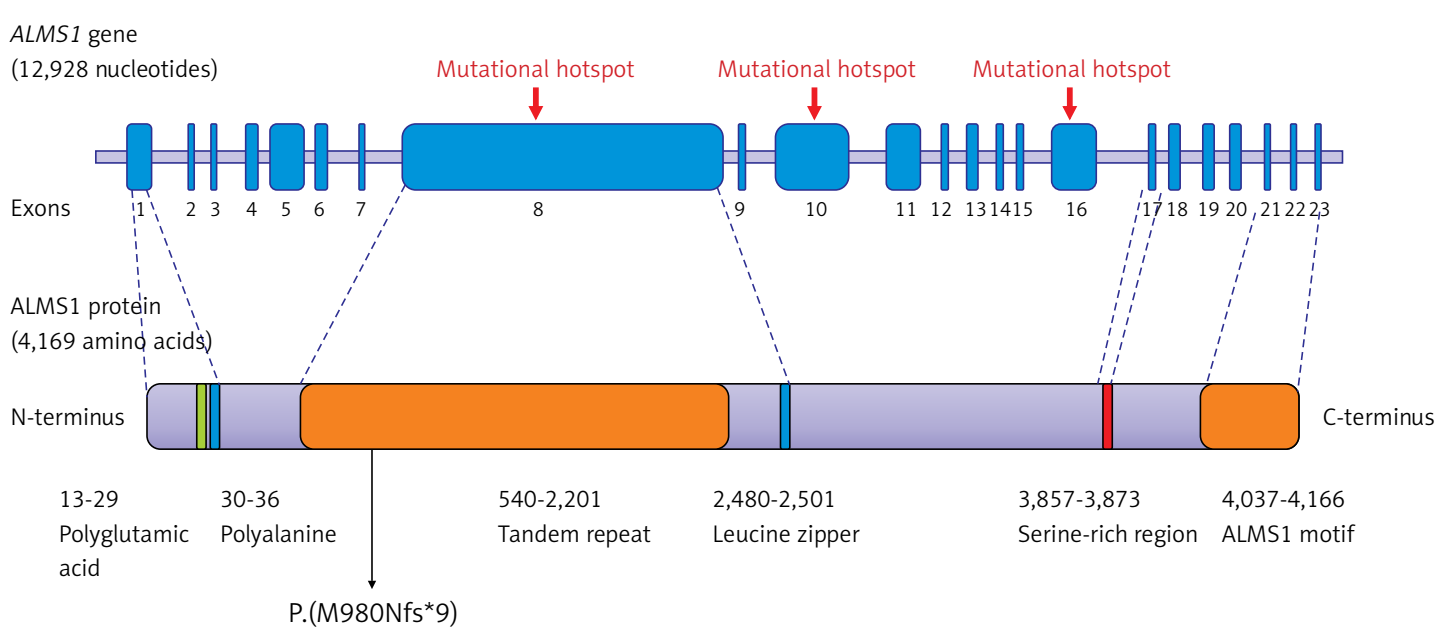

SLKNCLLFLD*

Human ALMS1 978 SLKMSAIPGLTDQKTVPTPTVPSGSFSHREKPSIFYQQEWPDS 1020

Figure 3. The graphic illustrates the distribution of 23 exons among 231,167 nucleotide sequence of the ALMS1 gene and the location of exon8: c.2938 dupA mutation that was present in the Saudi family

Exome Aggregation Consortium (ExAC), Greater Middle East (GME) Variome project, EURO-WABB (LOVD) and Saudi Human Genome Project (SHGP). The Mutation Taster webtool predicted this mutation as disease causing. The Sanger sequencing results also confirmed that both symptomatic brothers (II.2 \& II.5) were homozygous to the mutant allele, whereas their parents (I.1 \& I.2) were heterozygous and the asymptomatic members (II.1, II.4 \& II.6) were homozygous to the reference allele. These findings confirm that the ALMS1: c.2938 dupA mutation is inherited in the family members in the autosomal recessive mode (Figure 2). Sanger sequencing results for the candidate variant are shown in Table II and in Figure 4.

\section{Discussion}

In the present study, we described a novel mutation in the ALMS1 gene causative for Alström syndrome in one consanguineous family from Saudi Arabia. In this family, the proband (II.2) was admitted to the hospital for cardiac arrhythmias and seizures. Thus, he was initially suspected to have a cardiac arrythmia, but echocardiographic studies revealed a dilated cardiomyopathy with severe mitral valve regurgitation. He was born with bilateral astigmatism and retinal dystrophy and developed severe bilateral sensorineural hearing loss at puberty. He was found to have allergic rhinitis and hypertriglyceridemia at the age of 21 years. There was a history of obesity starting in childhood but no blood sugar abnormality. All these findings suggested a mitochondrial disorder. But Sanger sequencing is not an ideal strategy to screen all the mitochondrial genes as it requires lot of time and a series of laboratory experiments. Hence, we chose to perform WES, which is able to perform mutation scanning for both nuclear and mitochondrial genes in a single experiment. WES was performed for the proband (II:2) and his affected brother (II.5). After multiple filtration steps, we were left with a potentially disease-causing

Table II. Genotypes of variants in the ALMS1 gene as determined by Sanger sequencing

\begin{tabular}{|lclcc|}
\hline Family members & Genotype & Zygosity & Exon & Type/effect \\
\hline I.1 & GA/GAA & Heterozygous & $8 / 23$ & Normal \\
\hline I.2 & GA/GAA & Heterozygous & $8 / 23$ & Normal \\
\hline II.2 & GAA/GAA & Homozygous & $8 / 23$ & Frameshift variant \\
\hline II.4 & GA/GA & Homozygous & $8 / 23$ & Normal \\
\hline 11.5 & GAA/GAA & Homozygous & $8 / 23$ & Frameshift variant \\
\hline II.6 & GA/GA & Homozygous & $8 / 23$ & Normal \\
\hline
\end{tabular}


$(\mathrm{GA}>\mathrm{GAA})$

(Homozygous)

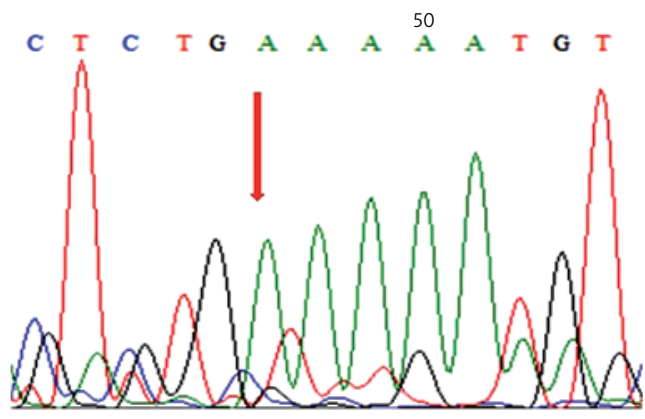

(Patient)
$(G A>G A)$

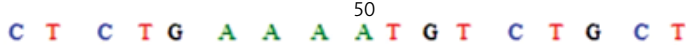

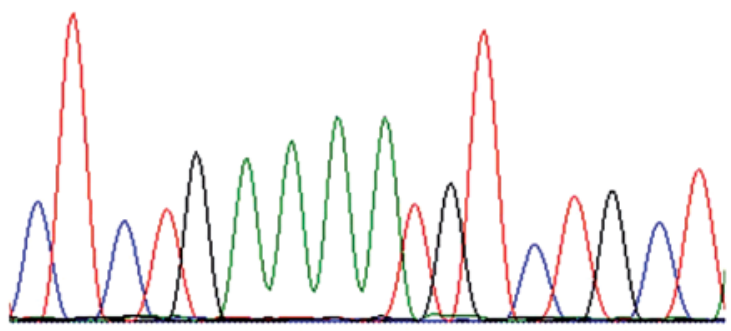

(Control)

Figure 4. Sanger sequencing chromatogram showing the site of the nucleotide duplication for the frameshift mutation (ALMS1: c.2938 dupA) found in the affected siblings and the wildtype sequence

variant, c.2938 dupA (p.Met980Asnfs*9) in the ALMS1 gene in exon 8 in both the proband and his brother. Both inherited the variant in an autosomal recessive manner.

The c.2938 dupA mutation is located on exon 8, which is an almost $6 \mathrm{~kb}$ long nucleotide sequence and encodes a huge tandem repeat sequence (TRS) domain, which makes up $40 \%$ of the coding region [17]. Accordingly, we postulate that this truncating ALMS1 protein at codon 980 , which is located in the TRS domain lying between 540 and 2201 amino acids, might generate unstable protein that undergoes nonsense mediated decay, leading to the loss of approximately $77 \%$ of the total 4169 amino acids of ALMS1 protein. Molecular defects of ALMS1 might lead to the failure of different molecular processes that are involved in the primary cilium function, cell cycle regulation, apoptosis, extracellular matrix production, adipogenesis, cell migration, cytoplasmic microtubular organization and endosomal transport $[13,18]$.

Alström syndrome patients are known to demonstrate variable clinical expressivity. This significant degree of variability, even within members of the same family or between families, generates complications for a universal definition $[1,19]$. Table III shows the detailed phenotype-genotype characteristics for all Alström syndrome patients from Saudi Arabian families. It displays the clinical manifestations associated with each mutation. Eye abnormality is the predominant clinical phenotype, which is observed in $100 \%$ of Alström syndrome patients, followed by obesity and DCM, which occur in $68 \%$ and $48 \%$ of cases, respectively. Thirty-seven percent of the cases presented with SNHL, while $26 \%$ had blood sugar abnormality [2, $4,7,20-22]$.

In our patients, the proband (II.2) and his affected brother (II.5) did not show typical symptoms, although they carry the same mutation. For example, both of them had vision abnormality and
DCM, but the affected brother (II.5) did not present with obesity or hearing loss, in contrast to the proband, who had both. In addition, the intra-familial variability of cardiac presentation between the two affected brothers is a noticeable finding as the proband was diagnosed with the DCM at the age of 22 years with subsequent recovery in the follow-up, in contrast to his brother, who developed DCM from birth and experienced a severe course of cardiac disease culminating in end stage heart failure and death. This may be explained by the earlier hypothesis that the genotype-phenotype relationship of ALMS1 gene mutations might be altered by other modifier genes or by environmental influences [9]. We also assume that the proband's sister, who died of complications of Wilms' tumor, also had the bi-allelic ALMS1 mutation. Since she died prior to the commencement of our study, we could not look for other overt symptoms in her. Still, this study highlights that renal diseases could be another feature of ciliopathies, and in vitro knockdown of the ALMS1 gene in mice was reported to cause stunted cilia on kidney epithelial cells [23]. However, the effect of ALMS 1 bi-allelic truncating mutation on the onset and progression of renal dysfunction in Alström syndrome is unknown. So, we speculate that the renal phenotype in the sister of the proband is due to ALMS1 mutation.

In conclusion, this study reports a novel and pathogenic ALMS1 mutation (c.2938 dupA) as causative of Alström syndrome. Future in vitro functional studies could ascertain its pathogenic impact on the protein structure and function. Our genotype-clinical phenotype findings indicate that the atypical presentation of Alström syndrome, even within siblings, could sometimes lead to clinical misdiagnosis. Hence, the present study emphasizes the utility of exome sequencing to support the clinical diagnosis of Alström syndrome patients. 


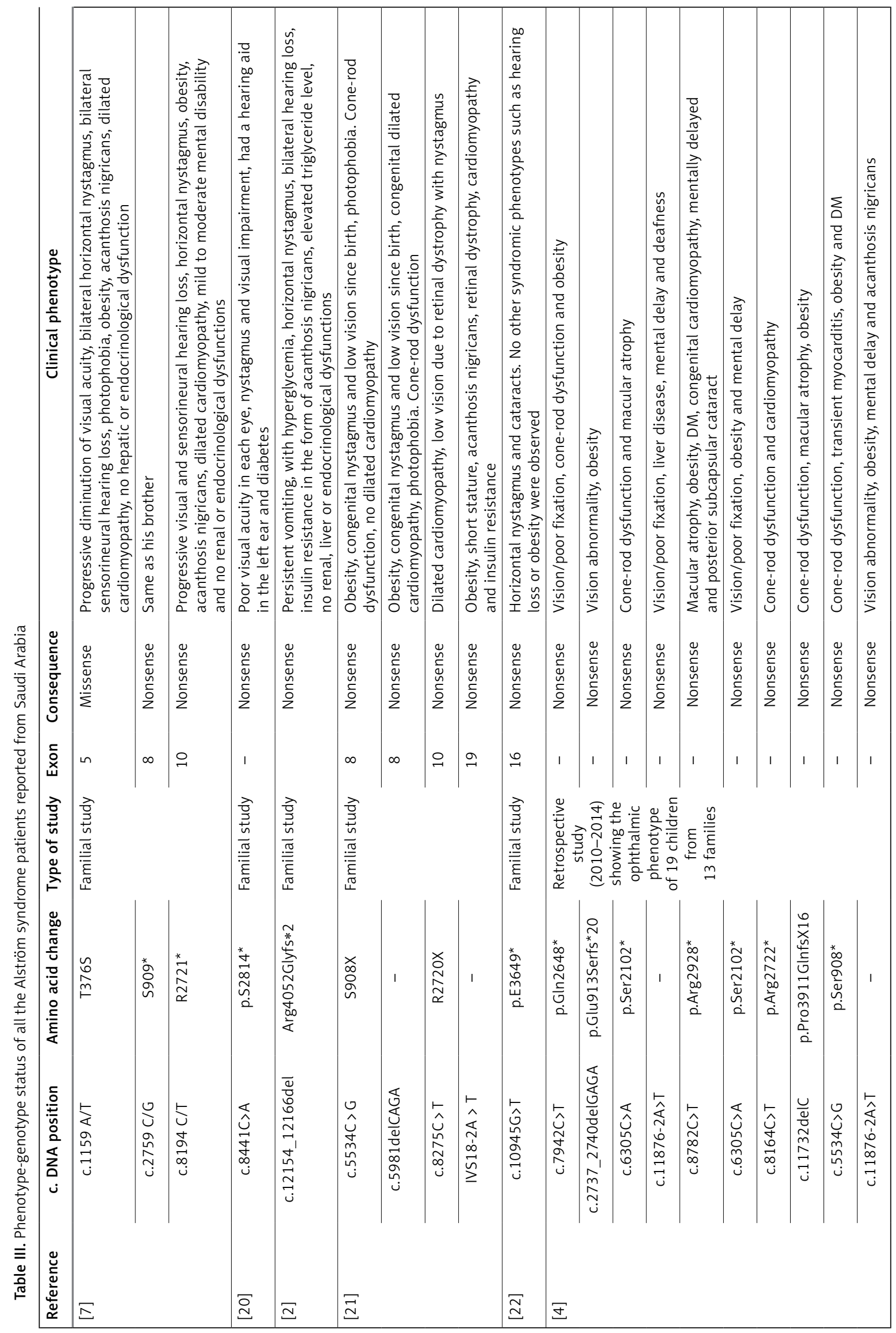


Amnah Yousuf Bdier, Faten Abdullah Al-Qahtani, Prashant Kumar Verma, Naeem Abdulmoneem Alshoaibi, Nuha Mohammed Alrayes, Noor Ahmad Shaik, Roger Sik Yin Foo, Zahurul Alam Bhuiyan, Jumana Yousuf Al-Aama

\section{Acknowledgments}

Amnah Yousuf Bdier and Faten Abdullah AlQahtani are shared first authors. This work was supported by a fund by King Abdulaziz City for Science and Technology (KACST), grant number 10-BIO1075-03. All authors sincerely thank Princess Al-Jawhara Al-Brahim Centre of Excellence in Research of Hereditary Disorders, King Abdulaziz University for providing the technical training and support to carry out this study.

\section{Conflict of interest}

The authors declare no conflict of interest.

\section{References}

1. Joy T, Cao H, Black G, et al. Alstrom syndrome (OMIM 203800): a case report and literature review. Orphanet J Rare Dis 2007; 2: 49.

2. Bakar AA, Kamal NM, Alsaedi A, Turkistani R, Aldosari D. Alström syndrome: a novel mutation in Saudi girl with insulin-resistant diabetes. Medicine 2017; 96: e6192.

3. Colunga Blanco S, Velasco Alonso E, Corros Vicente C, Martín Fernández M, García Pérez L, Morís de la Tassa C. Alström syndrome: a rare cause of cardiomyopathy. Rev Esp Cardiol 2018; 71: 296-8.

4. Khan AO, Bifari IN, Bolz HJ. Ophthalmic features of children not yet diagnosed with Alstrom syndrome. Ophthalmology 2015; 122: 1726-7.e2.

5. Poli L, Arroyo G, Garofalo M, et al. Kidney transplantation in Alström syndrome: case report. Transplant Proc 2017; 49: 733-5.

6. Paisey RB, Steeds R, Barrett T, Williams D, Geberhiwot T, Gunay-Aygun M. Alström syndrome. GeneReviews ${ }^{\circledR}$ [Internet] 2003 Feb 7 [Updated 2019 Jun 13]. In: Adam MP, Ardinger $\mathrm{HH}$, Pagon RA, et al., editors. GeneReviews ${ }^{\circledR}$ [Internet]. Seattle (WA): University of Washington, Seattle; 1993-2020. Available from: https://www. ncbi.nlm.nih.gov/books/NBK1267

7. Kamal NM, Sahly AN, Banaganapalli B, et al. Whole exome sequencing identifies rare biallelic ALMS1 missense and stop gain mutations in familial Alström syndrome patients. Saudi J Biol Sci 2020; 27: 271-8.

8. Arbustini E, Narula N, Dec GW, et al. The MOGE (S) classification for a phenotype-genotype nomenclature of cardiomyopathy: endorsed by the World Heart Federation. J Am Coll Cardiol 2013; 62: 2046-72.

9. Marshall JD, Muller J, Collin GB, et al. Alström syndrome: mutation spectrum of ALMS1. Hum Mutat 2015; 36: 660-8.

10. Braune K, Volkmer I, Staege MS. Characterization of alstrom syndrome 1 (ALMS1) transcript variants in hodgkin lymphoma cells. Plos One 2017; 12.

11. Astuti D, Sabir A, Fulton P, et al. Monogenic diabetes syndromes: locus-specific databases for Alström, Wolfram, and Thiamine-responsive megaloblastic anemia. Human Mutation 2017; 38: 764-77.

12. Hearn T. ALMS1 and Alström syndrome: a recessive form of metabolic, neurosensory and cardiac deficits. Jol Med 2019; 97: 1-17

13. Hearn T, Renforth GL, Spalluto C, et al. Mutation of ALMS1, a large gene with a tandem repeat encoding 47 amino acids, causes Alström syndrome. Nat Genet 2002; 31: 79-83.
14. Lindsey S, Brewer C, Stakhovskaya O, et al. Auditory and otologic profile of Alström syndrome: comprehensive single center data on 38 patients. Am J Med Genet C Semin Med Genet 2017; 173: 2210-8.

15. Zmyslowska A, Borowiec M, Antosik K, et al. Genetic evaluation of patients with Alström syndrome in the Polish population.Clin Genet 2016; 89: 448-53.

16. Al-Aama J, Alhashem A. An Overview of Medical Genetic Services in Saudi Arabia. 2018. Available at: http://www. cags.org.ae/contentfiles/uploads/files/Chapter\%202.pdf

17. Marshall JD, Maffei P, Collin G, Naggert J. Alstrom syndrome: genetics and clinical overview. Curr Genomics 2011; 12: 225-35.

18. Girard D, Petrovsky N. Alström syndrome: insights into the pathogenesis of metabolic disorders. Nat Rev Endocrinol 2011; 7: 77-88.

19. Chen JH, Geberhiwot T, Barrett TG, Paisey R, Semple RK. Refining genotype-phenotype correlation in Alström syndrome through study of primary human fibroblasts. Mol Genet Genomic Med 2017; 5: 390-404.

20. Safieh LA, Al-Otaibi HM, Lewis RA, Kozak I. Novel mutations in two Saudi patients with congenital retinal dystrophy. Middle East Afr J Ophthalmol 2016; 23: 139-41.

21. Aldahmesh MA, Abu-Safieh L, Khan AO, et al. Allelic heterogeneity in inbred populations: the Saudi experience with Alström syndrome as an illustrative example. Am J Med Genet A 2009; 149A: 662-5.

22. Wang $X$, Wang $H$, Cao $M$, et al. Whole-exome sequencing identifies ALMS1, IQCB1, CNGA3, and MYO7A mutations in patients with Leber congenital amaurosis. Hum Mutat 2011; 32: 1450-9.

23. Li G, Vega R, Nelms K, et al. A role for Alström syndrome protein, alms1, in kidney ciliogenesis and cellular quiescence. PLoS Genet 2007; 3: e8. 